\title{
Force generation of bio-inspired hover kinematics
}

\author{
Ruben B.R. Vandenheede* \\ Delft University of Technology, Delft, Zuid-Holland, 2600AA, Netherlands \\ Luis P. Bernal ${ }^{\dagger}$ and Christian L. Morrison ${ }^{\ddagger}$ \\ University of Michigan, Ann Arbor, MI, 48105, United States \\ Sean Humbert $\$$ \\ University of Maryland, College Park, MD, 20742, United States
}

\begin{abstract}
This paper presents the results of an experimental study of the aerodynamics of an elliptical flap plate wing in pitch-plunge motion. Several wing motion kinematics are derived from the kinematics of the Agrius Convolvuli (hawk moth) in hover. The experiments are conducted at a Reynolds number of 4,800 and reduced frequency of 0.38 , which are typical of the hawk moth flight. Three cases are reported: the hawk moth kinematics in which the elevation angle is ignored, the hawk moth kinematics with a correction to account for elevation angle effects, and a harmonic pitch-plunge kinematic of the same frequency and amplitude as the hawk moth kinematics. In all cases the wing pivots about the leading edge. The experiments are performed in The University of Michigan water channel. The wing model used has a Zimmerman planform shape with aspect ratio 3.87. Phase averaged force measurements are reported. Average thrust coefficients of 2.79, 2.64 and 2.39, respectively, are measured for the three cases. The measured peak thrust coefficients are 5.0, 4.8 and 6.1, respectively. The propulsion figure of merit in hover was also measured and found to be $0.47,0.48$ and 0.49 for the three cases. The flow evolution was measured using PIV. The results show formation of Leading Edge Vortices (LEV) and Trailing Edge Vortices (TEV) at different phases of the motion which depend on the particular kinematics. The relation between LEV and TEV vortex evolution and force generation is discussed.
\end{abstract}

\section{Nomenclature}

$A \quad$ Area swept by the wing, $\mathrm{m}^{2}$

$A R$ Aspect ratio, -

$C_{L} \quad$ Lateral force coefficient, -

$C_{T} \quad$ Thrust coefficient, -

$F_{\text {lateral }}$ Lateral force, $\mathrm{N}$

$L_{\text {ref }}$ Reference length

$M \quad$ Figure of merit, -

$M_{x} \quad$ Moment around the axis $\mathrm{x}, \mathrm{Nm}$

$P \quad$ Power, $\mathrm{W}$

$R \quad$ Semi-span, $\mathrm{m}$

Re Reynold number

$T$ Thrust force, $\mathrm{N}$

$U$ Velocity

$\Phi \quad$ Flapping amplitude, rad

c $\quad$ Mean chord length, $\mathrm{m}$

\footnotetext{
*Graduate Research Assistant, Department of Aerospace Engineering, vdh.ruben@gmail.com

$\dagger$ Associate Professor, Department of Aerospace Engineering, lpb@umich.edu

${ }^{\ddagger}$ Graduate Research Assistant, Department of Aerospace Engineering, cmorrisz@umich.edu

$\S$ Assistant Professor, Department of Aerospace Engineering, humbert@umd.edu
} 
$f \quad$ Flapping frequency, $\mathrm{Hz}$

$h \quad$ Plunge position experiment

$h_{0} \quad$ Plunge amplitude experiment

$k \quad$ Reduced frequency

$t^{*} \quad$ Normalized time $t / T$

$v \quad$ Flow velocity

$\alpha \quad$ Feathering angle, rad

$\delta \quad$ Pitch angle, rad

$\nu_{a} \quad$ Viscosity of air

$\nu_{w} \quad$ Viscosity of water

$\phi \quad$ Flapping angle

$\rho \quad$ Density

$\theta \quad$ Elevation angle

$\omega \quad$ Vorticity

- Denotes the $1^{\text {st }}$ time derivative

- Denotes an averaged or normalized quatity

\section{Introduction}

Recent developments in micro air vehicles are revolutionizing remote sensing and information gathering. For very small vehicles, flapping wings have unique characteristics that could provide exceptional capabilities in maneuverability, hover performance, as well as being able to operate in confined spaces. These performance characteristics are observed in insects and small birds motivating studies of the aerodynamics of flapping wings of biological flyers. ${ }^{10}$

Biological flyers demonstrate unusual maneuverability necessary for very small MAVs. Understanding of the aerodynamics of biological flyers can provide insights useful for the design of flapping wing MAVs. A very well studied example of a biological flapping flyer is the hawk moth. ${ }^{11}$ Hawk moths show consistent wing beat parameters for an individual as well as comparing different animals. Also the main features, dominant in insect flapping flight, are present in the stroke of the hawk moth, without the complications of extra mechanisms present in a number of other insects, e.g. clap \& fling mechanisms and exaggerated ventral flexion.

The wings of insects produce more lift than predicted with conventional aerodynamic theory, i.e.compared to the steady motion at the same velocity and angle of attack. Aerodynamic studies of hawk moths and other small insects have shown relevant phenomena in the generation of force, e.g. LEV vortex formation and wake capturing. ${ }^{3}$ The LEV can account, in part, for the quantitative disagreement with the forces predicted by conventional aerodynamic theories, ${ }^{4}$ i.e. LEV generates a lower pressure area at the leading edge resulting in a large force production.

Recently the aerodynamics of pitch and plunging airfoils has received considerable attention (e.g. Yeon et al, ${ }^{2}$ Granlund et $\mathrm{al}^{5}$ and Rausch et $\left.\mathrm{al}^{9}\right)$. Granlund et $\mathrm{al}^{5}$ considered the aerodynamics of a flat plate that is free to pivot about the leading edge between $\pm 45^{\circ}$ incidence limiters and the translation is a prescribed sinusoidal function. For an aspect ratio of 3.4 they found that the stroke to chord ratio is the main factor affecting the thrust production, with small stroke to chord ratios evincing the lowest thrust. They found that the results are insensitive to changes in Reynolds number, within the 5,000 to 20,000 range. Furthermore they found no evidence of wake capture in these experiments.

In the present work we extend the pitch-plunge studies by considering the aerodynamics of an elliptical flap plate wing in pitch plunge motion with bio-inspired hover motions. The kinematics are derived from the hover kinematics of the hawk moth. It is impossible to capture all features from a three degree of freedom flapping motion into a two degree of freedom pitch-plunge motion. Therefore two main kinematics and a third purely sinusoidal motion are investigated. The first motion ignores the effect of the elevation angle and the second motion incorporates a correction for the loss of elevation angle. The experiments are initially conducted at the Reynolds number and reduced frequency of the hawk moth in hover, i.e. 4, 800 and 0.38 respectively. The force generation, propulsion efficiency and flow evolution are measured and reported. These results complement related work on the effects of flapping reported by Morrison et al ${ }^{8}$ and Yeo et al. ${ }^{13}$ 


\section{Brief description of the experimental setup}

The experiments are performed in The University of Michigan, Aerospace Engineering Department, water channel. The test section measures 610 by $610 \mathrm{~mm}$ and is $2.44 \mathrm{~m}$ long. The channel is capable of producing very low turbulence flow, the free turbulence intensity is less than $1 \%$. Flow speeds can range from 5 to $40 \mathrm{~cm} / \mathrm{s}$. For this experiment there is no flow in the channel, as we are investigating a hover case.

The wing has a chord of $79.4 \mathrm{~mm}$, a span of $241.3 \mathrm{~mm}$ and is cut out of an acrylic plate with a thickness of $2.75 \mathrm{~mm}$. The planform shape consists of two ellipses with the major axis equal to the span and the midpoint of the ellipse is the quarter chord position, cf.Figure 1a. The wing is clamped and mounted on a rig with two motors, hanging vertically in the water channel, as shown in Figure 1b. The motors are capable of performing pitch-plunge motions with a high degree of accuracy.

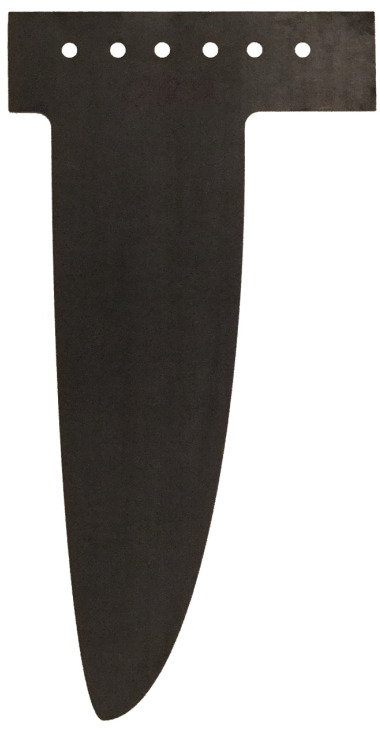

(a) Wing planform

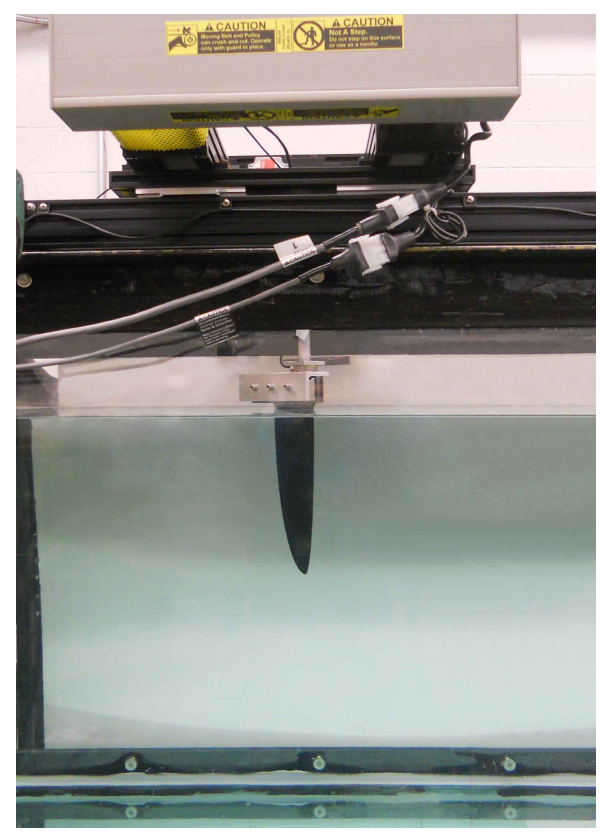

(b) Wing vertically mounted in the channel

Figure 1: Experimental Setup

The force data is acquired using the ATI Mini 40 force/torque sensor mounted on top of the wing clamp. The raw sensor data is then calibrated and filtered using a Butterworth low-pass filter to reduce noise.

The Particle Image Velocymetry (PIV) setup consists of a pulsed Nd:YAG laser (Spectra-Physics PIV300), an optical setup to form the laser sheet, an external timing system (a BNC 555 pulse delay generator and the Stanford Research Systems' DG535) and a high resolution 14bit cooled CCD camera system (Cooke, PCO4000) with a Nikon Micro-NIKKOR $105 \mathrm{~mm}$ lens. The camera has a sensor of 4008 by 2672 pixels.

The plunge amplitude is about $130 \mathrm{~mm}$. To obtain good spatial resolution three camera positions are used and the data is stitched together in the post processing routine. Therefore the camera is mounted on a slide and shifted to 3 positions that have an overlap region. The magnification of the imaging system is 14 pixels per $\mathrm{mm}$ and the field of view is 6225 by 3525 pixels (or 437 by $247 \mathrm{~mm}$ ). Images are acquired over 100 cycles and the data is phase averaged.

For the PIV data acquisition the flow is seeded with Titanium(IV) oxide, rutile powder with a diameter of approximately 5 micrometer. A dispersant, ammonium polymethacrylate (DARVAN C-N), is added to facilitate the suspension of the particles for longer periods of time. 


\section{Determining the relevant scaling and non-dimensional parameters}

The hover kinematics are mainly inspired by a Hawk moth, the Agrius Convolvuli. As a starting point for determining the relevant scaling and non-dimensional parameters, the values of the Agrius Convolvuli are used.

\section{A. Hawkmoth, Agrius Convolvuli}

The Agrius Convolvuli is a subspecies of the Hawk moth, Sphingidae. It is a large species with a wingspan of about $80-105 \mathrm{~mm}$. Similarly to hummingbirds, they have evolved to feed on nectar while hovering in front of a flower. This capability makes them an excellent study object for flapping hovering flight.

The kinematics of this moth in hovering flight are well known, cf. Section IV. The motion is relatively simple and does not include a clap and fling mechanism. The Agrius Convolvuli is four-winged, but the motion of the wings on both sides in hover is nearly in unison and can be assumed to act as one wing. The wing planform of both wings together is similar to the Zimmerman planform and will be modeled as such.

The parameters determining the dynamics are presented in Table 1. The flapping frequency and amplitude are those of the moth in hover.

Table 1: Agrius Convolvuli parameters

\begin{tabular}{|l|l|}
\hline Parameter & $\begin{array}{l}\text { Value Agrius } \\
\text { convolvuli }\end{array}$ \\
\hline Mean chord length $c_{m}$ & $18.3 \mathrm{~mm}$ \\
Semi-Span $R_{m}$ & $50.5 \mathrm{~mm}$ \\
Aspect ratio $A R_{m}$ & 2.76 \\
Flapping frequency $f_{m}$ & $26.1 \mathrm{~Hz}$ \\
Flapping amplitude $\Phi$ & $2 \mathrm{rad}$ \\
\hline
\end{tabular}

In order to calculate the relevant dimensionless parameters for hover, a reference point on the wing needs to be chosen. In general the wing tip is used for this purpose, ${ }^{12}$ however since the objective is to capture the most relevant phenomena and try to represent these in a pitch-plunge motion, the tip velocity will make the scaling parameters too large. A reasonable approximation is to use three quarters of the span $\left(L_{r e f}=0.75 R_{m}\right)$ as the reference radius.

$$
U_{\text {ref }}=2 \Phi f L_{r e f}
$$

The Reynolds number can then be rewritten as follows.

$$
R e=\frac{U_{r e f} c_{m}}{\nu_{a}}=\frac{2 \Phi f_{m} L_{r e f} c_{m}}{\nu_{a}}
$$

In turn the reduced frequency, equation 3 , is based on the same reference velocity.

$$
k=\frac{\pi f_{m} c_{m}}{U_{\text {ref }}}
$$

Based on three quarters of the span the Reynolds number and reduced frequency are 4,800 and 0.38 respectively.

\section{B. Relevant parameters experiment}

The experiment is a pure pitch-plunge motion, hence the reference velocity is equal to the average velocity during half of the period, cf. equation 4, depending only on the amplitude and frequency of the motion.

$$
U_{\text {ref }}=4 f h_{0}
$$


Substitution of the reference velocity, equation 4, leads to the following expression for the reduced frequency of the experiment. Note that in the pitch plunge case, for a certain wing, the reduced frequency is solely dependent on the amplitude of the motion.

$$
k=\frac{\pi c}{4} \frac{1}{h_{0}}
$$

Treating the reduced frequency as a constant, i.e. using the value of the Agrius Convolvuli, yields an expression for the amplitude of the motion.

$$
h_{0}=\frac{\pi c}{4 k}=129,1 \mathrm{~mm}
$$

Similarly the Reynolds number can be written as.

$$
R e=\frac{4 c}{\nu_{w}} f h_{0}
$$

Using the Reynolds number of the moth, an expression for the frequency of the experiment can be obtained.

$$
f=\frac{\operatorname{Re} \nu_{w}}{4 c h_{0}}
$$

This equation can be used directly to determine the frequency. However it is convenient to further develop the equation in a slightly different form. First, substitution of equation 6 yields.

$$
f=R e \frac{\nu_{w} k}{\pi c^{2}}
$$

Secondly, using equation 3 , we obtain.

$$
f=\operatorname{Re} \frac{\nu_{w}}{\pi c^{2}} \frac{\pi c_{m}}{2 \Phi R_{m}}
$$

Third, substitution of equation 2 leads to the following expression,

$$
f=\frac{2 \Phi f_{m} R_{m} c_{m}}{\nu_{a} c^{2}} \frac{\nu_{w} c_{m}}{2 \Phi R_{m}}
$$

which simplifies to.

$$
f=f_{m}\left(\frac{c_{m}}{c}\right)^{2} \frac{\nu_{w}}{\nu_{a}}
$$

Hence, from equation 12 it can be seen that the frequency of the experiment depends solely on the flapping frequency of the moth, the ratio of the mean chords squared and the ratio of the kinematic viscosities. The frequency of the present experiment is $0.15 \mathrm{~Hz}$.

$$
f=0.57 \% f_{m}=0.15 H z
$$

\section{Hover kinematics}

The goal is to perform a pitch-plunging motion in a water channel. The kinematics of the experiment are inspired on the kinematics of the hawk moth.

\section{A. Coordinate system Agrius Convolvuli}

The coordinate system used to describe the motion is shown in Figure 2a. Three angles are used to describe the position of the wing: ${ }^{11}$ the flapping angle $\phi$, the feathering angle $\alpha$ and the elevation angle $\theta$.

The elevation and flapping angle are defined positive as shown in Figure 2a and the feathering angle, $\alpha$, is negative in Figure 2a. 


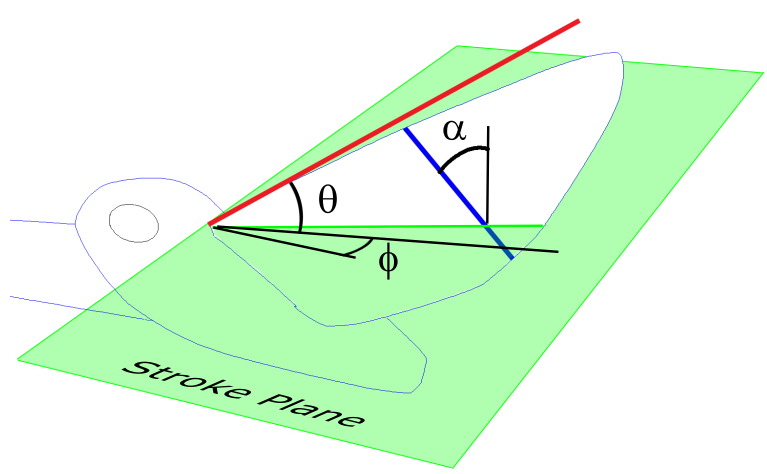

(a) Coordinate system for the motion kinematics

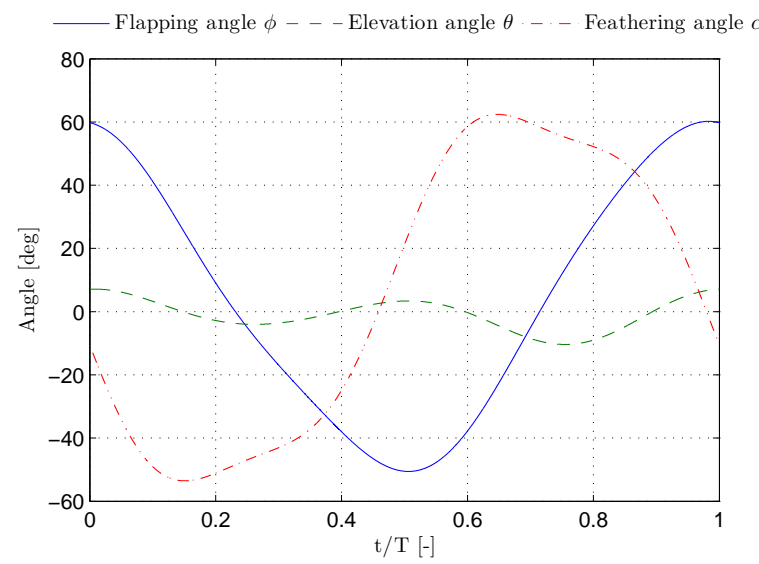

(b) Representation of the kinematic angles

Figure 2: Representation of the Agrius Convoluli kinematics

\section{B. Hover kinematics Agrius Convolvuli}

The kinematics of the Agrius Convolvuli are described by three angles. For the hover-case these angles vary in time as shown in Figure 2b, cf. $^{1}$

\section{Making the motion symmetric}

The three dimensional hover motion of the Agrius Convolvuli is made symmetric in order to convert it to a pure pitch-plunge motion at a later stage. This is done while capturing the most important features during hover, e.g. phase shift of feathering and flapping angle.

\section{Flapping angle}

The flapping angle has an average angle of about $4.0^{\circ}$, first the data is shifted to yield a mean angle of $0^{\circ}$. This is justified since this is just a matter of shifting the reference point.

Second, the down stroke is $2.3 \%$ longer than the upstroke, this small difference will be neglected, i.e. the upstroke will be slightly stretched in time to match the down stroke. The resulting angles are shown in Figure 3a, observe the small difference between down stroke angle and the up stroke here shown inverted to highlight the differences.

In a last step both half periods are averaged to make the motion completely symmetric, resulting in the averaged flapping angle in Figure 3a.

\section{Feathering angle}

A similar procedure is applied to the feathering angle. In this case there is no difference in duration of the up and down stroke. The values are shifted by $4.8^{\circ}$ to obtain a $0^{\circ}$ mean value. After this procedure the feathering angles during upstroke are nearly analogues to the angles during down stroke. Both up and down stroke are averaged, and the result is shown in Figure 3b.

\section{Elevation angle}

The elevation angle is also made symmetric, the same procedure as for the feathering angle is used. The mean is shifted from about $-1.0^{\circ}$ to $0^{\circ}$ and both half periods are then averaged, shown in Figure 3c. 


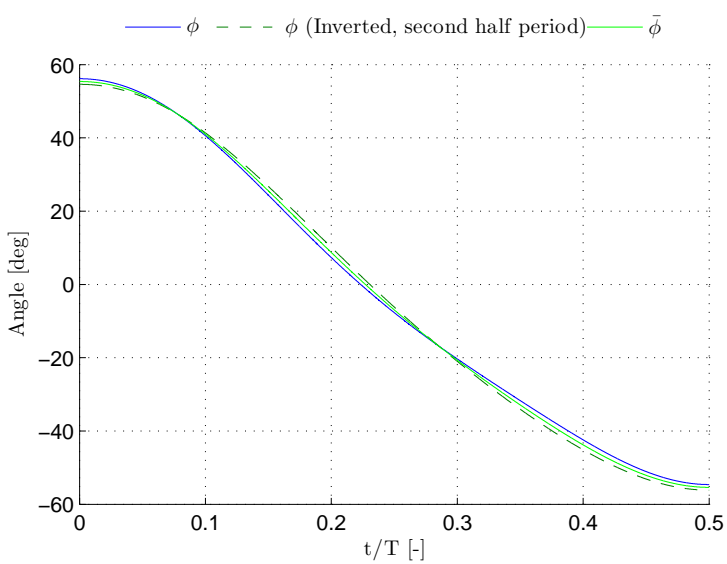

(a) Adjustments of the flapping angle $\phi$

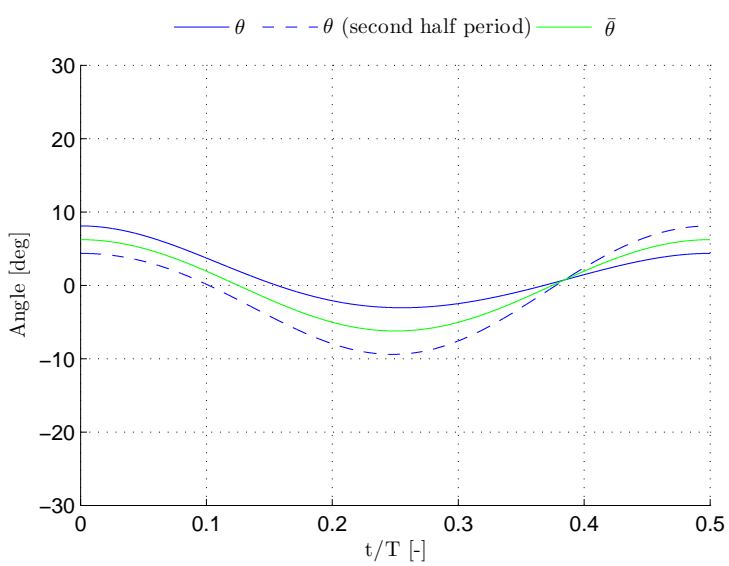

(c) Adjustments of the elevation angle $\theta$

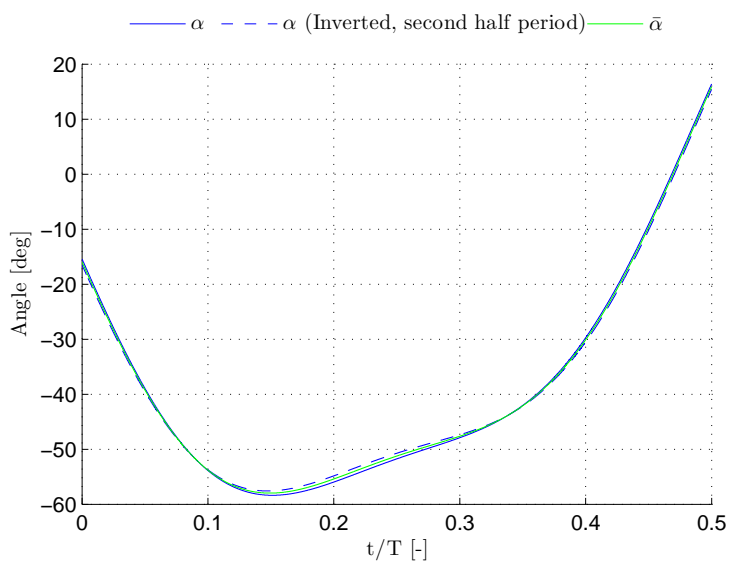

(b) Adjustments of the feathering angle $\alpha$

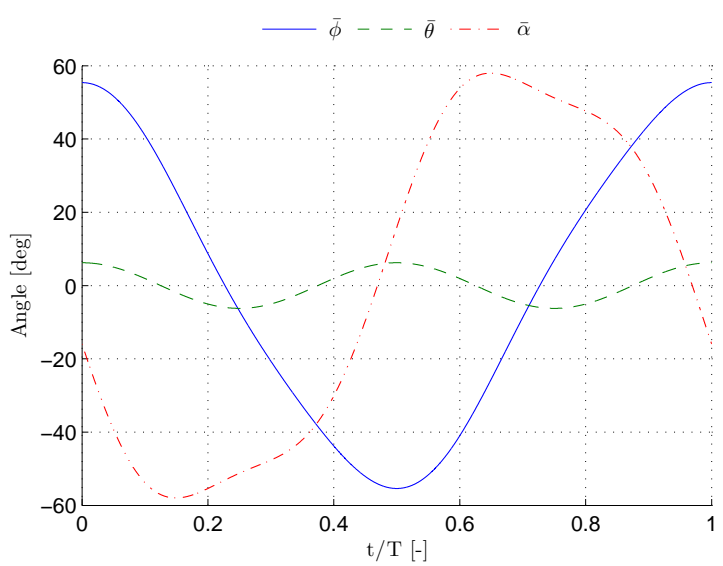

(d) Agrius Convoluli hover kinematics made symmetric

Figure 3: Making the motion symmetric

\section{Adjusted kinematic angles}

A summary of the resulting, symmetric angles after adjustment is shown in Figure 3d.

\section{Hover kinematics experiment}

There are a number of different ways convert the hawkmoth kinematics to the pitch-plunge kinematics for the present experiment. The main goal is to preserve the most important features in the conversion from flapping motion to a pure pitch-plunge motion. For instance the phase lag between feathering angle and flapping angle is assumed to be important and has been retained. to examine the effect of other parameters two different motions are investigated.

\section{Ignoring the elevation angle}

The first way of determining the kinematics would be to simply ignore the elevation angle and using the feathering angle and the flapping angle.

The flapping angle translates to a plunging motion, $h(t)$, as follows. 


$$
h(t)=\frac{\bar{\phi}(t)}{\Phi} h_{0}
$$

The pitch angle, $\delta(t)$, is than equal to the feathering angle. The rotation point for this case coincides with the leading edge at the root of the airfoil.

$$
\delta(t)=\bar{\alpha}(t)
$$

\section{Compensating feathering and flapping angle for the loss of elevation angle}

Instead of ignoring the elevation angle, it can also be incorporated in the pitch-plunge motion by changing the reference frame, i.e. computing the effective pitch angle relative to the direction of motion. This process is illustrated in Figure 4.

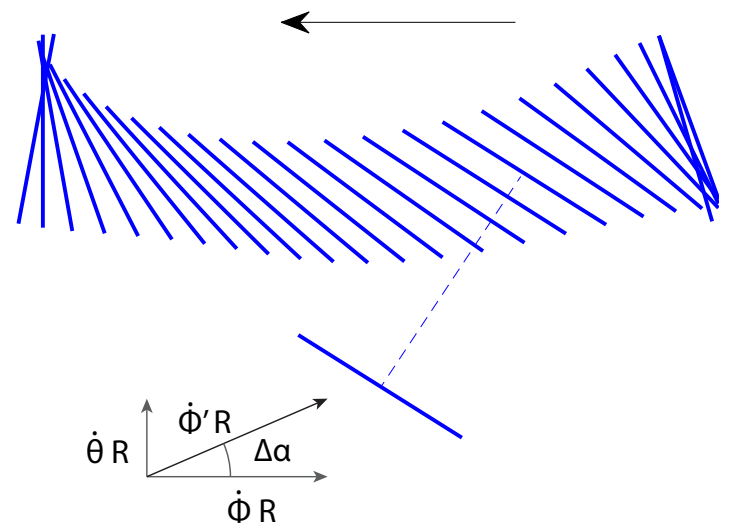

Figure 4: Converting a flapping motion to a pitch-plunge motion

The change in pitching angle, $\Delta \alpha$, due to the elevation angle can be quantified as follows.

$$
\Delta \alpha=-\arctan \left(\frac{\dot{\bar{\theta}}}{\dot{\bar{\phi}}}\right)
$$

Yielding equation 17 as the expression for the corrected feathering or pitch angle. The variation in time of the pitch angle is plotted in Figure 5a. The rotation point coincides with the leading edge.

$$
\begin{gathered}
\delta(t)=\bar{\alpha}(t)-\arctan \left(\frac{\dot{\bar{\theta}}(t)}{\dot{\bar{\phi}}(t)}\right) \\
U_{\text {eff }}= \pm \sqrt{(\dot{\bar{\theta}} R)^{2}+(\dot{\bar{\phi}} R)^{2}}
\end{gathered}
$$

The sign is determined by the sign of the angular flapping speed, $\dot{\phi}$. The effective velocity can be written as $U_{\text {eff }}=\dot{\phi}^{\prime} R$, yielding.

$$
\dot{\phi}^{\prime}= \pm \sqrt{\dot{\bar{\theta}}^{2}+\dot{\bar{\phi}}^{2}}
$$

Through integration and the correct determination of the integration constant the compensated flapping angle is found, and it is plotted in Figure 5b. Converting the elevation angle to an equivalent plunge position $h(t)$.

$$
h(t)=\frac{\phi^{\prime}(t)}{\Phi} h_{0}
$$



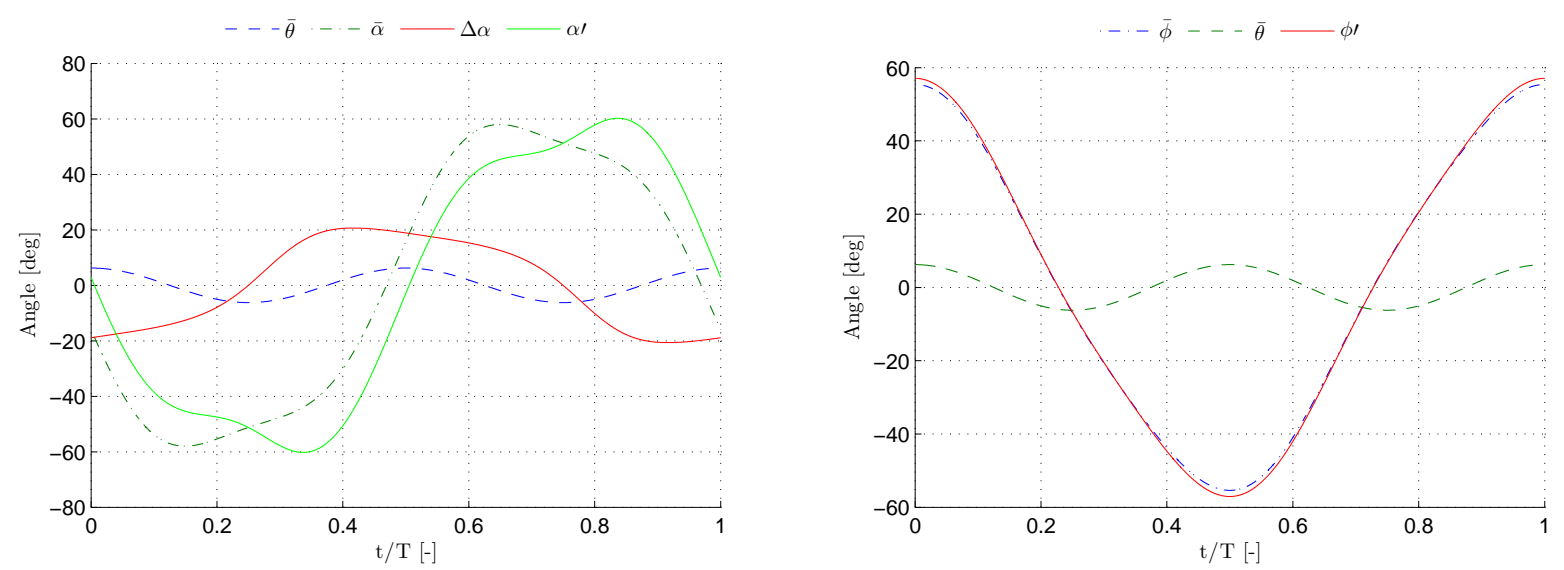

(a) Compensation of the feathering angle for the loss of elevation(b) Compensation of the flapping angle for the loss of elevation angle angle

Figure 5: Compensation for the loss of elevation angle

\section{Resulting kinematics}

Three motions result, the first hover motion ignores the loss of elevation angle and simply converts the flapping and feathering angle. Shown in Figure 6a. This motion will be referred to as hover motion 1 or HM1.

The second hover motion, Figure 6b, incorporates a correction in the flapping and feathering angle for the elevation angle. This motion will be referred to as hover motion 2 or HM2.

As a third reference, a pure sinusoidal motion has been created with the same pitch and plunge amplitude as the basic Agrius Convolvuli kinematics. The sinusoidal kinematics are plotted in Figure 6c.

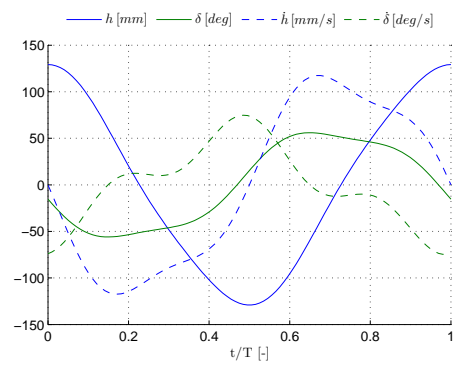

(a) Pitch-plunge hover motion experiment ignoring the loss of elevation angle (hover motion 1)

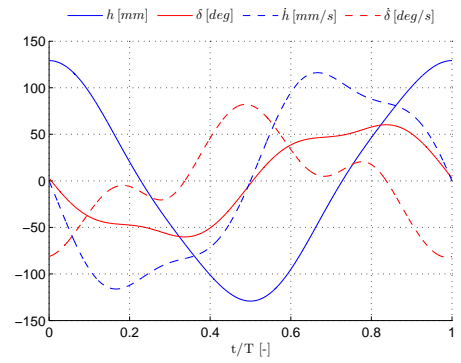

(b) Pitch-plunge hover motion experiment with compensation for the loss of elevation angle (hover motion 2)

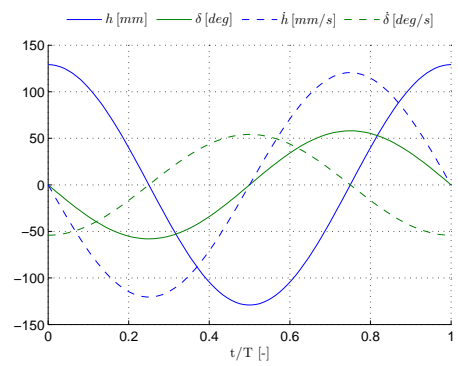

(c) Pure Sinusoidal pitch-plunge motion with the same amplitude and frequency as the Agrius Convolvuli

Figure 6: Resulting kinematics

\section{Force data acquisition}

\section{A. Data acquisition process}

For each motion kinematics, force data is acquired in 6 separate sets of 25 cycles. The first five cycles are cropped from each measurement eliminating any start up phenomena that might occur. The data is then calibrated and converted to thrust and lateral force components. The thrust is defined as the force perpendicular to the plunge motion, similarly the lateral force is the force lateral to the plunge motion. 
The thrust and lateral force components are filtered with a low pass Butterworth filter with a cut off frequency of $3 \mathrm{~Hz}$. The attenuation properties of the filter are shown in Figure 7 . To eliminate the phase shift introduced by the filter, it is run over the data back and forth. This process ensures zero phase distortion and squares the magnitude of the original filter.

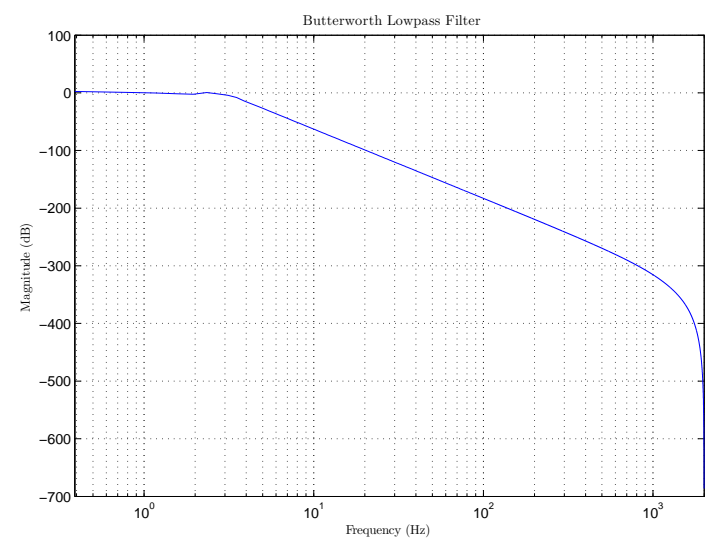

Figure 7: Butterworth low pass filter attenuation

Next the remaining 120 cycles are phase averaged and the sample mean and the corresponding standard deviation are calculated.

\section{B. Non-dimensionalization of the data}

The resulting force data can be non-dimensionalized by the dynamic pressure (based on the reference velocity $U_{\text {ref }}$ equal to $4 f h_{0}$, Section III B) and the projected wing surface area. The thrust coefficient is then defined as shown in equation 21.

$$
C_{T}=\frac{T}{q S}
$$

Similarly the lateral force coefficient is normalized by the same dynamic pressure and the projected wing surface area.

To obtain a sense of efficiency of the thrust production, the ideal power is calculated using simple momentum theory. ${ }^{7}$

$$
P_{\text {ideal }}=\sqrt{\frac{T^{3}}{2 \rho A}}
$$

Where $T$ is the average thrust produced, $\rho$ is the density of the fluid and $A$ represents the area swept by the wing. The power input can be calculated by multiplying the angular speed with the corresponding moment and the translational velocity with the corresponding force, as shown in equation 23.

$$
P=F_{\text {lateral }} \dot{h}+M_{z} \dot{\delta}
$$

A convenient figure of merit, $M$, is then defined as follows.

$$
M \equiv \frac{P_{\text {ideal }}}{P}
$$




\section{Results base motions}

In this section the results of the base motions, described in Section IV D, are presented.

For hover motion 1, the thrust coefficient, as shown in Figure 8a, peaks at 4.7. The peak occurs at $20 t^{*}$ just after the pitch angle and the plunge speed reach their maximum value. The average thrust coefficient is 2.79. The non-dimensionalized standard deviation of the mean varies between 0.60 and 0.17 or between $4 \%$ and $13 \%$ of the maximum thrust. The average of the absolute value of the lateral force coefficient is 3.00 , as shown in Figure 8d. The lateral force coefficient increases fairly rapid and varies around a value larger than the average. Three local maxima exist 3.2, 3.3 and 4.8 respectively. The figure of merit for this motion is 0.47 .

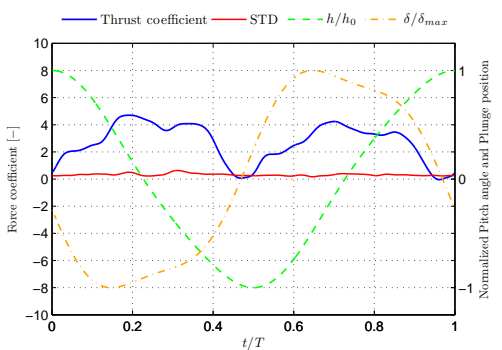

(a) Thrust coefficient hover motion 1

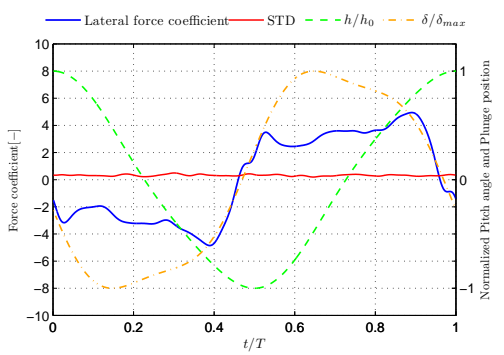

(d) Lateral force coefficient hover motion 1

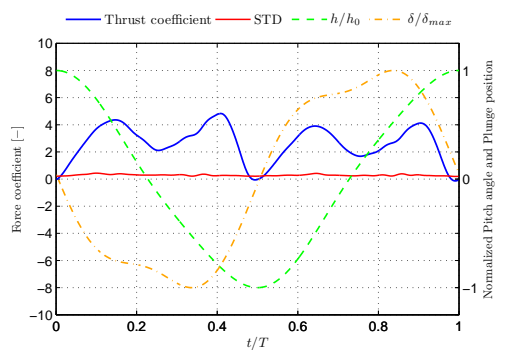

(b) Thrust coefficient hover motion 2

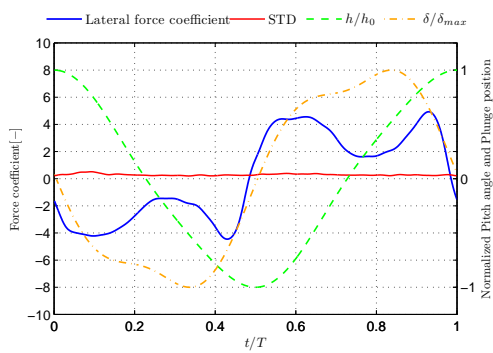

(e) Lateral force coefficient hover motion 2

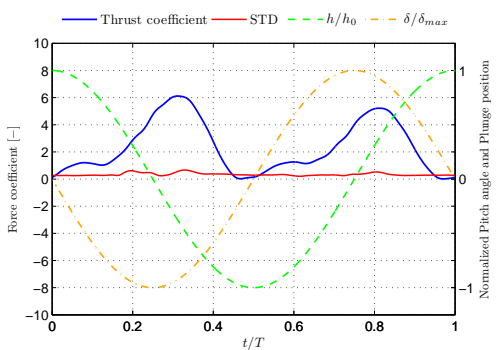

(c) Thrust coefficient pure sinusoidal motion

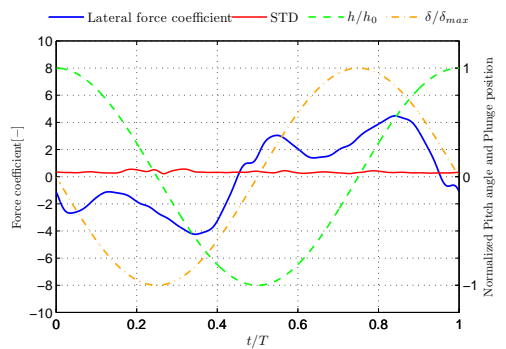

(f) Lateral force coefficient pure sinusoidal motion

Figure 8: Hover motions force coefficients

Two peaks exist in the thrust coefficient for hover motion 2, 4.4 and 4.8 respectively. The location of the first maximum occurs at $15 t^{*}$ and the second maximum occurs at 41 percent of the period. The local minima occur at the end of the stroke and right in the middle, at $\left.t^{*}=25\right)$, their values are -0.06 and 2.12. The average thrust coefficient equates to 2.64. The non-dimensionalized standard deviation of the mean varies between 0.43 and 0.19 or less then $10 \%$ of the maximum thrust coefficient. The absolute values of the lateral force coefficient average at 2.98 , the shape consist of two peaks with a local minimum in between. The peaks are located at 10 and $43 t *$ with values of 4.22 and 4.45 . The figure of merit equals 0.48 .

The sine motion thrust coefficient also has two peaks, the first peak is relatively small (about 1.2) and the second peak is substantially larger, 6.11 at $32 t^{*}$. The average thrust coefficient is 2.39 . The standard deviation of the mean varies between 0.67 and 0.21 or between 3 and $11 \%$ of the maximum thrust coefficient. The absolute lateral force coefficient averages at 2.39. Resulting in a figure of merit of 0.49.

The force on the wing is primarily perpendicular to the airfoil, this can also be seen in Figure 8, where the pitch angle is zero the thrust force is also zero. By means of comparison all force coefficients of the different motions are plotted in Figure 9. The average coefficients are tabulated in Table 2. It can be concluded that the figures of merit are all in the same order of magnitude. HM1 produces the most thrust, about $6 \%$ 
more than HM2 and 17\% more than the pure sinusoidal motion. As expected, for all motions the average lateral force coefficient (in absolute values) is of the same order of magnitude as the average thrust coefficient.

Assuming a number of hawk moths variable in weight and size ${ }^{6,12}$ the average thrust coefficient needed for hover would be around 0.88 to 1.01 (using the average velocity at $75 \%$ span position). The thrust coefficients for the motions in the experiment calculated using equation 21 are $2.79,2.64$ and 2.39 respectively, which is of the same order of magnitude. There are several factors increasing the thrust coefficient of the pitch plunge motion compared to the flapping motion. The speed of the wing at the root is not zero, which leads to a large contribution to the force since at the root the chord is the largest. The average speed over the airfoil is larger since it moves in its entirety, rather than just flapping. And last the swept area is larger for the pitch plunging motion compared to the flapping motion.

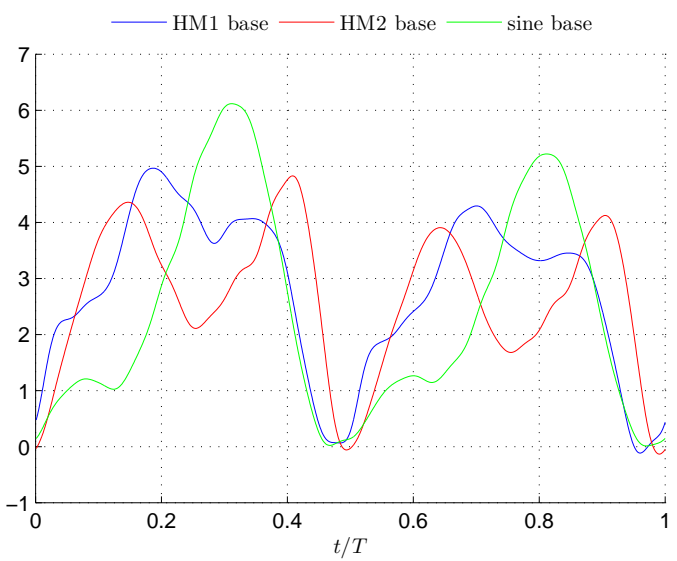

(a) Thrust coefficient

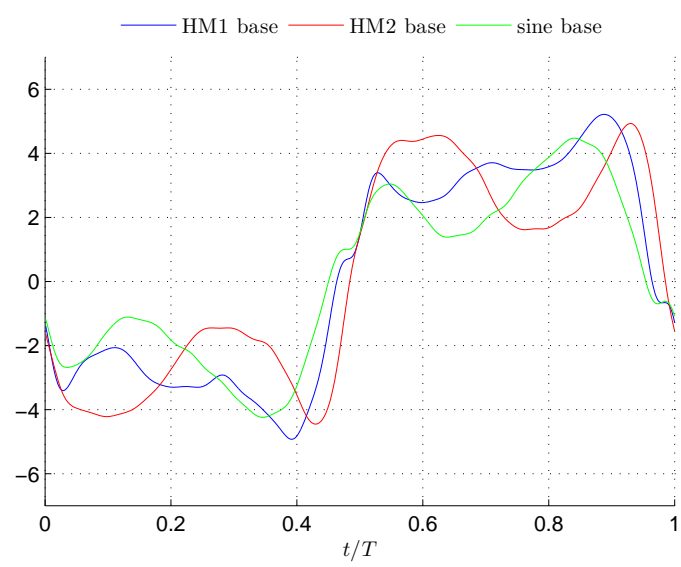

(b) Lateral force coefficient

Figure 9: Base motion force coefficients

Table 2: Base motion average force coefficients

\begin{tabular}{c|ccc} 
& HM1 & HM2 & Sine \\
\hline$C_{T}$ & 2.79 & 2.64 & 2.39 \\
$\left|C_{\text {Lateral }}\right|$ & 3.00 & 2.98 & 2.39 \\
$M$ & 0.47 & 0.48 & 0.49
\end{tabular}

\section{Particle image velocimetry}

The particle image velocimetry (PIV) data documents the flow topology. Using flow visualization we saw a significant axial flow and a large contraction of the wake. Therefor the measurements are taken at half span with a $2 \mathrm{~ms}$ time delay between the images and the resolution as described in Section II. These settings give us a good estimate of the flow topology, while keeping the measurements reliable both in the vortices close to the airfoil as further away from the airfoil where the flow speeds are much smaller, in the order of 0.7 to $0.1 U_{\text {ref }}$.

For all vorticity plots the vorticity is normalized with the reference velocity, the average tip speed, and the mean chord, shown in equation 25.

$$
\bar{\omega}=\frac{\omega c}{U_{\text {ref }}}
$$

In the middle of the vorticity plots the thrust and lateral force coefficients are plotted, vertical lines in 
this plot depict the phases, labeled $a$ through $f$, at which the PIV images are taken.

In the velocity plots, the magnitude of the vectors is only to scale with the other vectors in the same plot. To give an estimate of the magnitude the maximum velocity for each vertical line of vectors is given at the bottom of each plot. The maximum measured velocity is normalized by the reference velocity, as depicted in equation 26.

$$
\bar{v}=\frac{v}{U_{\text {ref }}}
$$

The axes in all plots show the distance in mean chords, with zero being the position of the leading edge at half span and the mid position of the plunge motion. Note that the chord length at half span is larger than the mean chord length. The plots are ordered chronologically from left to right, top to bottom. In all figures the airfoil is plunging downwards or the plunge velocity is zero, the leading edge points to the left side of the figure.

Figure 10 shows the phase-averaged vorticity and streamlines of the phase-averaged flow field for hover motion 1 at half span and phases as indicated. At the beginning of the stroke we see that the LEV from the previous half stroke breaks into two pieces, a small part flowing over the leading edge dissipating almost immediately and a larger part moves along the airfoil surface to the trailing edge where it interacts with the TEV or starting vortex. There is evidence of one LEV forming from approximately $0.2 t^{*}$ until the end of the stroke. The largest thrust coefficient occurs at $t^{*}=0.2$. After the change in pitch rate at $t^{*}=0.4$, where the largest force coefficient occurs, another starting vortex appears at the trailing edge. This vortex and the starting vortex at stroke reversal, which has opposite circulation, combine to form a persistent vortex structure.

The phase-averaged vorticity and streamlines for hover motion 2 are plotted in Figure 11. Similarly to HM1, the LEV breaks into two parts at stroke reversal. The larger part joins the starting vortex at the trailing edge. The other part remains around the leading edge and is entrained and annihilated by the new LEV having opposite circulation. In this case the LEV detaches between $t^{*}=0.2$ to 0.4 , stimulated by the increase of the pitch angle at $t^{*}=0.25$. A new LEV forms around $t^{*}=0.4$. These flow features correlate closely with the two peaks in force coefficients.

In Figure 12, the phase-averaged vorticity and streamlines are plotted for the sine motion. At the start of the stroke during stroke reversal, the vorticity contours show significantly weaker LEV and TEV vortices compared to the other two kinematics, which is consistent with the relative low force coefficient measured at these phases. For this kinematics the LEV does not form until around $t^{*}=0.2$. In this case the force coefficients at the beginning of the stroke are small but as the LEV grows larger the force coefficients surmount the maximum coefficients of both HM1 and 2. There is no formation of a TEV at the stroke reversal, in contrast to HM1 and 2.

In general, the PIV data shows the time history of the flow topology differs distinctly. The vortical flow evolution is very different comparing the three motions. This was also reflected in the time history of the forces, although the average force coefficients are of the same order of magnitude, i.e. within a $20 \%$ range with respect to each other. 


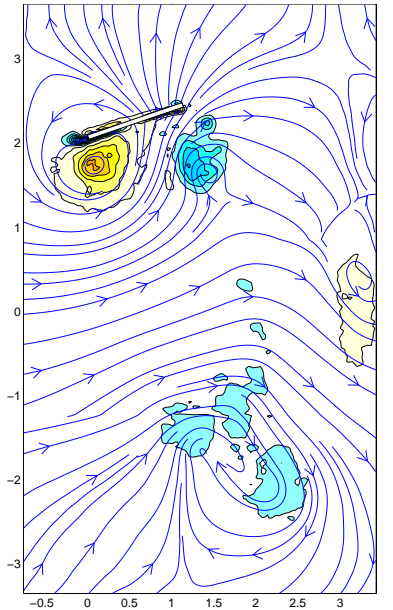

(a) $t^{*}=0.0, C_{T}=0.47, C_{\text {Lat }}=$ $-1.39$

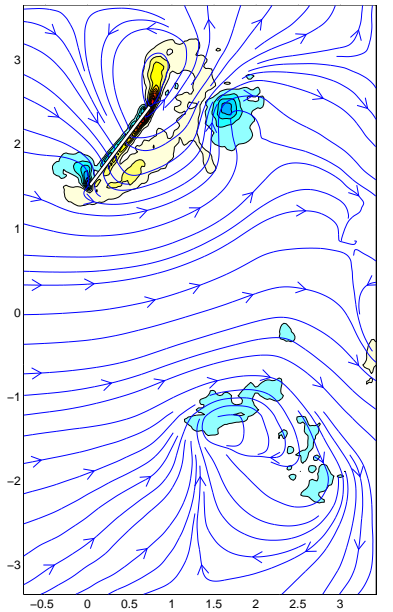

(b) $t^{*}=0.1, C_{T}=2.68$, $C_{\text {Lat }}=-2.11$

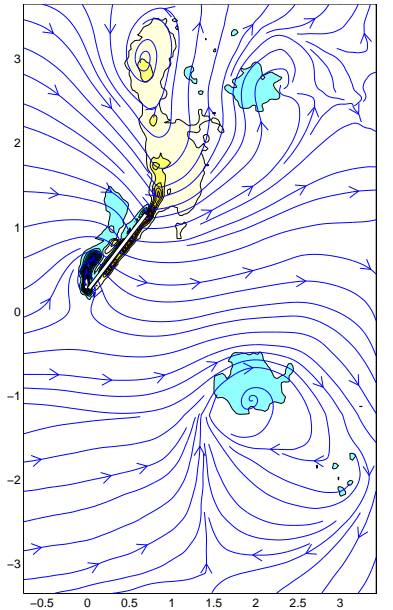

(c) $t^{*}=0.2, C_{T}=4.90, C_{\text {Lat }}=$ $-3.30$
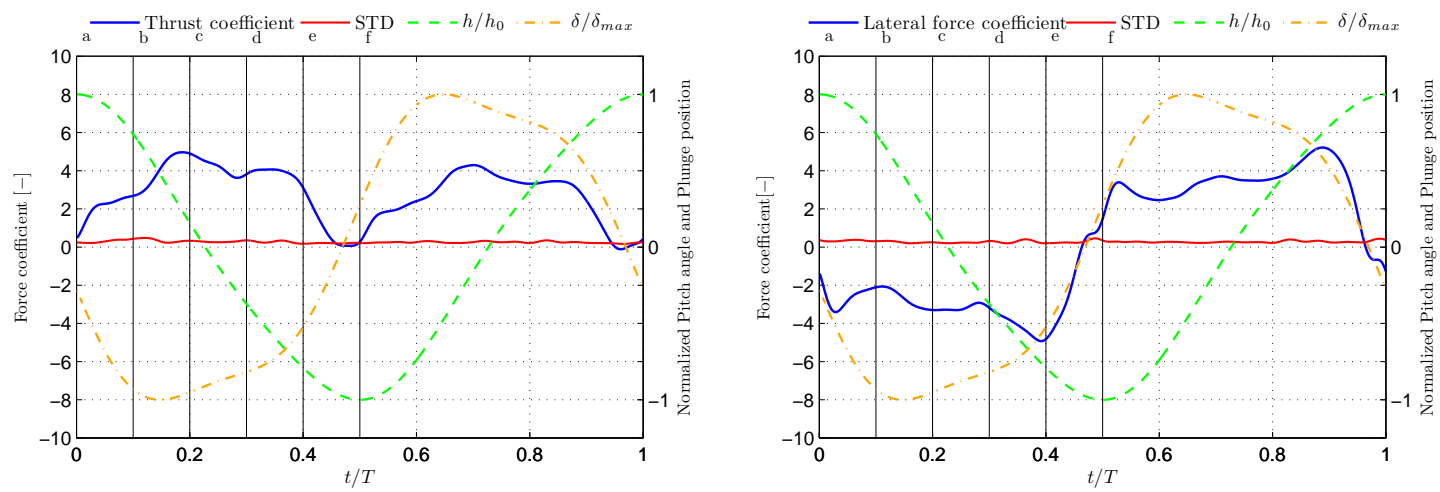

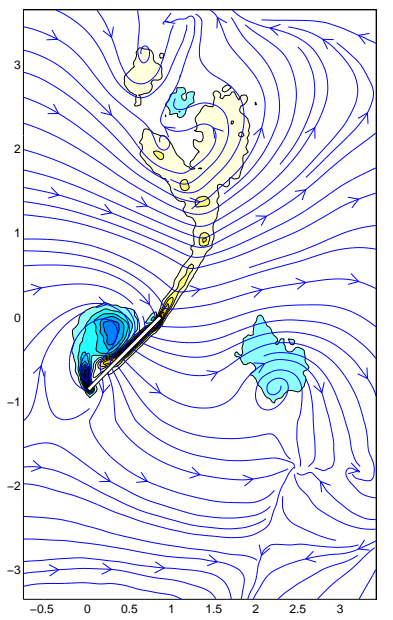

(d) $t^{*}=0.3, \quad C_{T}=3.82$, $C_{\text {Lat }}=-3.16$

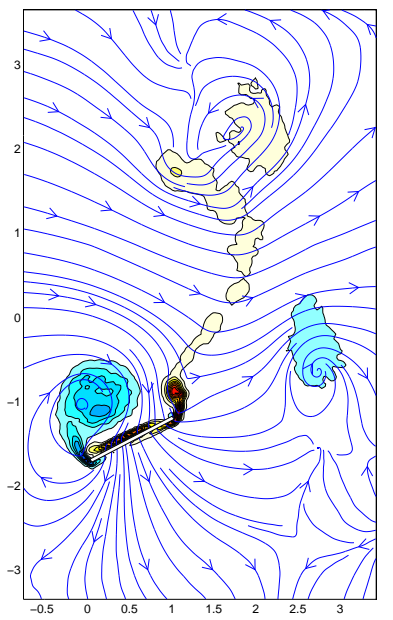

(e) $t^{*}=0.4, C_{T}=3.11, C_{\text {Lat }}=$ $-4.82$

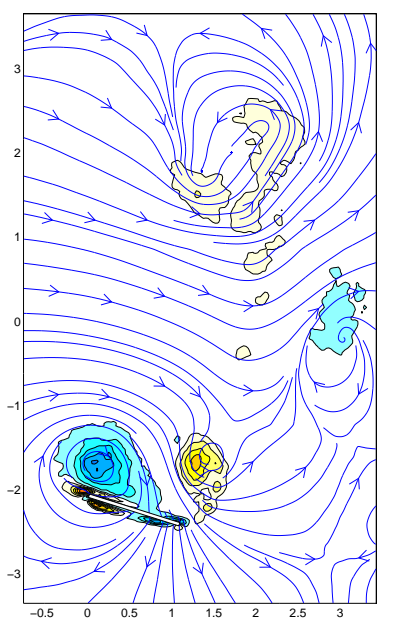

(f) $t^{*}=0.5, C_{T}=0.27, C_{\text {Lat }}=$ $-1.47$

Figure 10: Vorticity and streamline plots for HM1 base at half span, $C_{T}=2.79$ and $\left|C_{\text {Lat }}\right|=3.00$ 


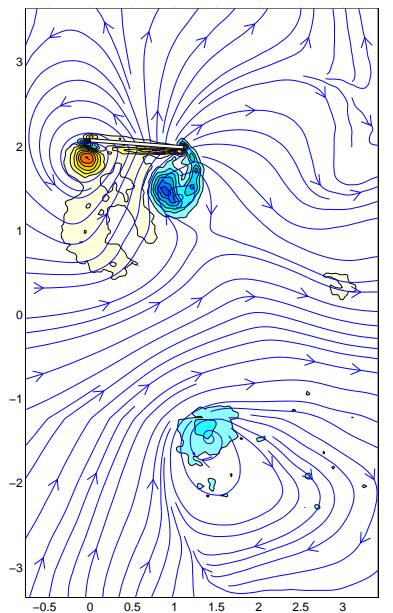

(a) $t^{*}=0.0, C_{T}=-0.03$, $C_{\text {Lat }}=-1.64$

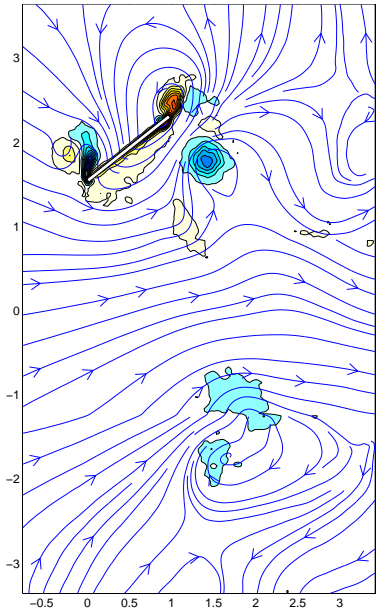

(b) $t^{*}=0.1, \quad C_{T}=3.70$, $C_{\text {Lat }}=-4.23$

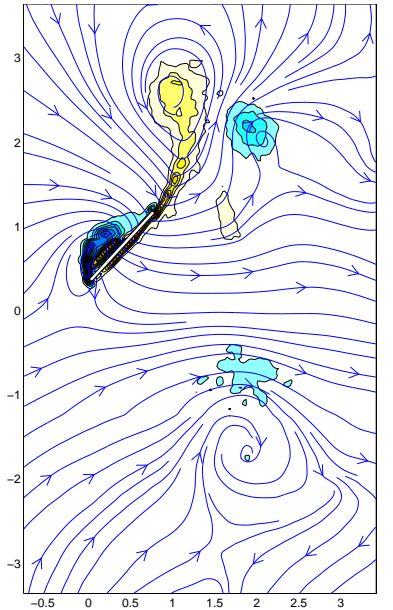

(c) $t^{*}=0.2, C_{T}=3.29, C_{\text {Lat }}=$ $-2.76$
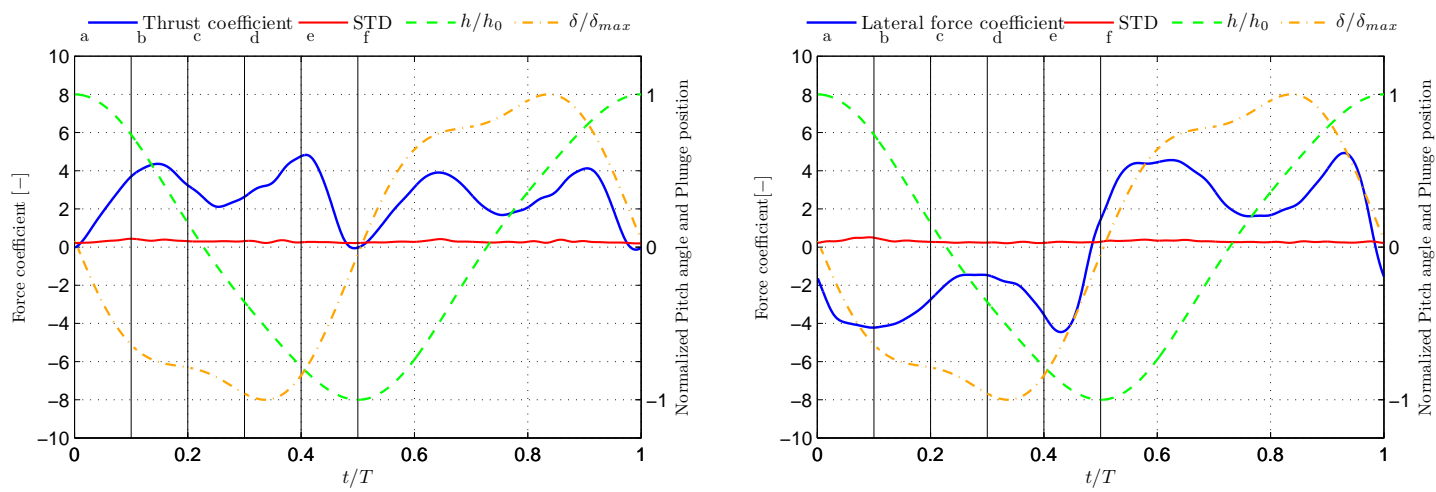

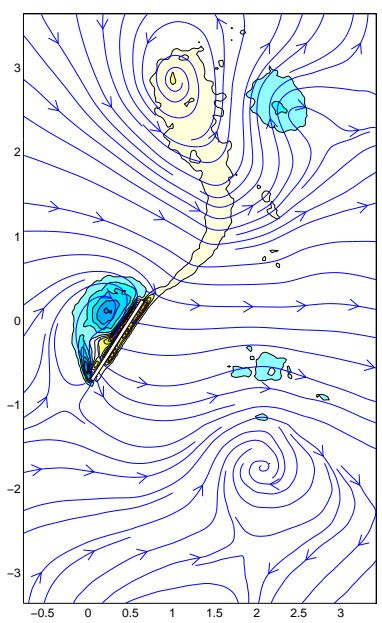

(d) $t^{*}=0.3, C_{T}=2.61$, $C_{\text {Lat }}=-1.46$

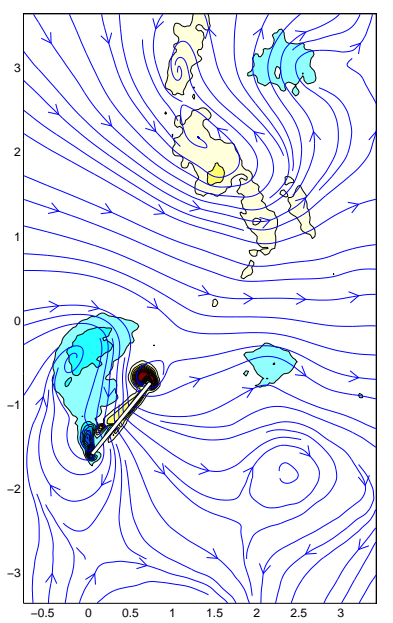

(e) $t^{*}=0.4, C_{T}=4.76, C_{\text {Lat }}=$ $-3.57$

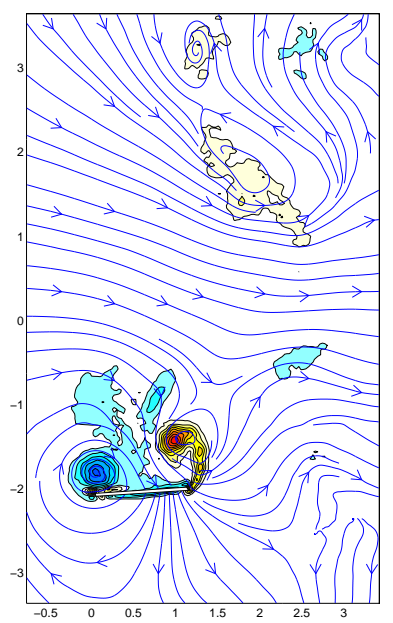

(f) $t^{*}=0.5, C_{T}=-0.06$, $C_{\text {Lat }}=1.43$

Figure 11: Vorticity and streamline plots for HM2 base at half span, $C_{T}=2.64$ and $\left|C_{\text {Lat }}\right|=2.98$ 


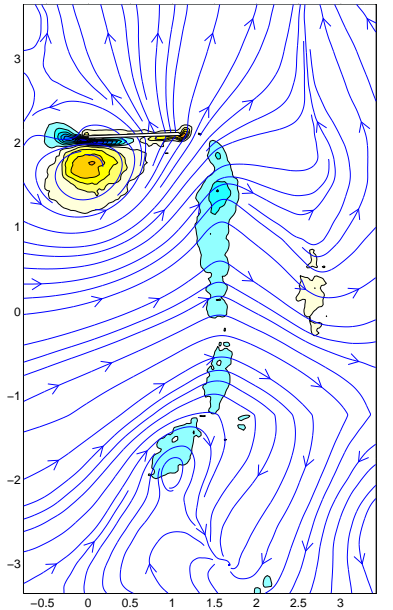

(a) $t^{*}=0.0, C_{T}=0.18, C_{\text {Lat }}=$ $-1.19$

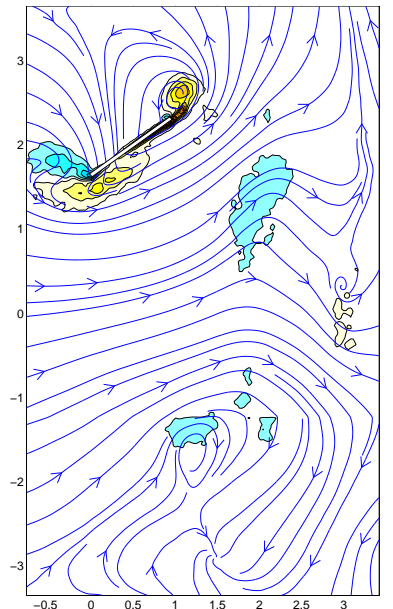

(b) $t^{*}=0.1, \quad C_{T}=1.16$ $C_{\text {Lat }}=-1.50$

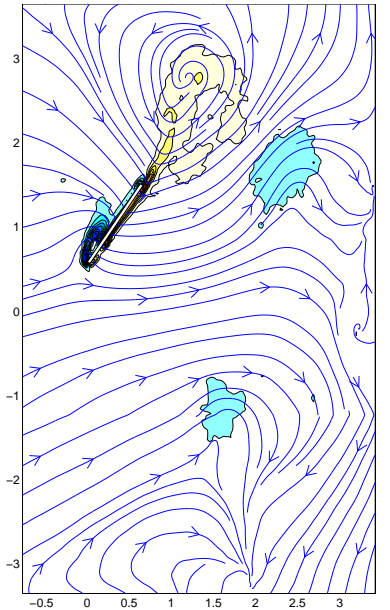

(c) $t^{*}=0.2, C_{T}=2.90, C_{\text {Lat }}=$ $-1.82$
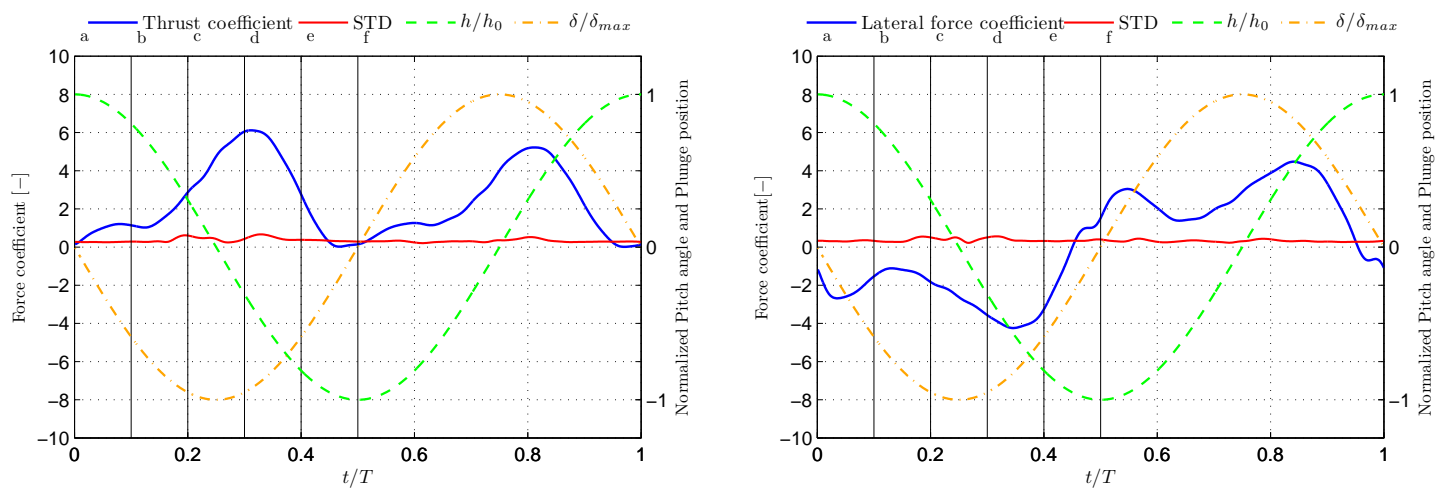

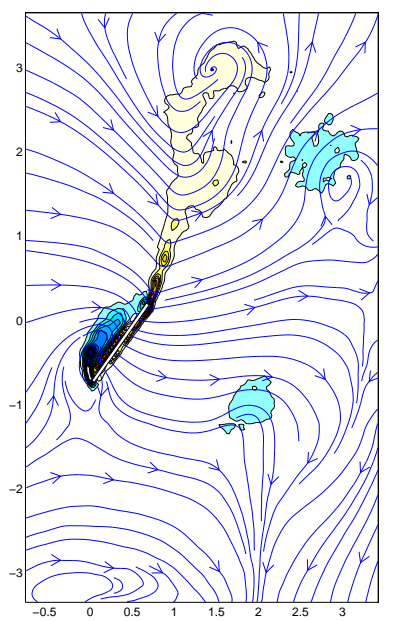

(d) $t^{*}=0.3, \quad C_{T}=6.06$ $C_{\text {Lat }}=-3.58$

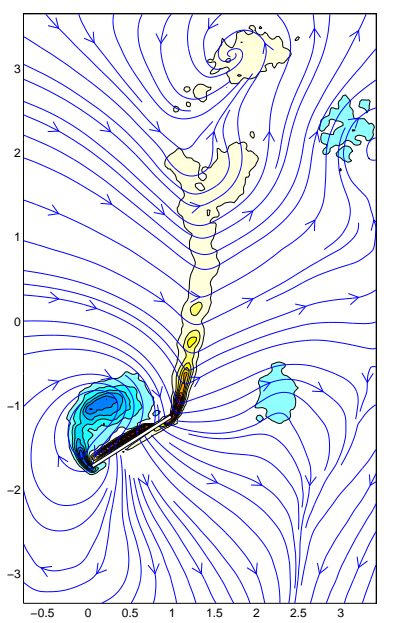

(e) $t^{*}=0.4, C_{T}=2.75, C_{\text {Lat }}=$ $-3.26$

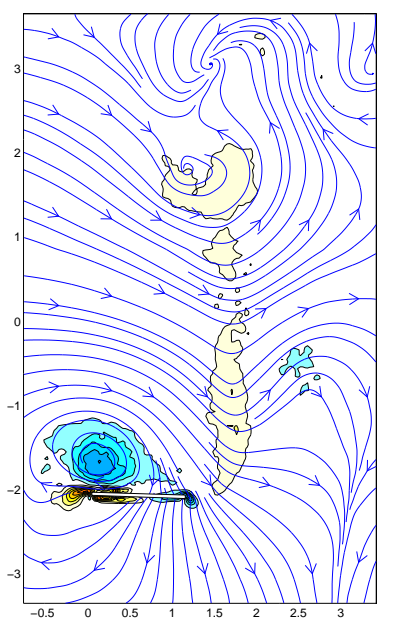

(f) $t^{*}=0.5, C_{T}=0.16, C_{\text {Lat }}=$ 1.51

Figure 12: Vorticity and streamline plots for Sine base at half span, $C_{T}=2.39$ and $\left|C_{\text {Lat }}\right|=2.39$ 


\section{Summary and conclusions}

There are a number of ways to convert a flapping motion to a pitch and plunge motions. In this paper two ways of converting the flapping motion of the Agrius Convolvuli to a pitch-plunge motion are presented and tested. The first motion, HM1, ignores the loss of elevation angle and simply translates the flapping angle into a plunge motion by using a reference speed at $75 \%$ of the span and uses the feathering angle as the pitch angle. The second motion is similar but incorporates a correction for the loss of elevation angle by adding the elevation angle to the feathering angle as a kind of effective angle of attack. A third motion is a pure sinusoidal motion with the same Reynolds number and reduced frequency, i.e. 4,800 and 0.38 respectively (cf. Agrius Convolvuli).

The thrust production and its efficiency have been measured For HM1, HM2 and Sine the average thrust coefficients are 2.79, 2.64 and 2.39 respectively. For all motion the force coefficients on average are larger than the thrust coefficient of a real moth needed to hover, but they are of the some order of magnitude. There are several factors increasing the thrust coefficient of the pitch plunge motion compared to the flapping motion. The speed of the root is non-zero, which leads to a big contribution since here the chord is at its largest. The average speed over the airfoil is larger since it moves in its entirety, rather than just flapping. And last the swept area is larger for the pitch plunging motion compared to the flapping motion.

Hover motion one produces more thrust than hover motion two and the pure sinusoidal motion. The difference between HM1 and HM2 are small, the pure sinusoidal motion produces roughly $14 \%$ less thrust. The force history plots differ strongly from one another, indicating a unique vortical flow evolution comparing the three cases. Hover motion one produces a more constant thrust coefficient, i.e. compared to the other motions it has a less steep gradient overall. The force history of hover motion two shows two peaks where the force coefficient is approcimately 4 . For the purely sinusoidal motion the force coefficient has a very small peak in the beginning of the stroke and a large peak near $0.3 t^{*}$ where the force coefficient is approximately 6. The propulsion efficiencies are all of the same order of magnitude, approximately 0.48 .

The PIV data reveals detailed information about the flow evolution. For HM1 there is one leading edge vortex formed during the entire stroke. Per half cycle there two TEV's created, one at the beginning and one right before stroke reversal. The second hover motion, HM2, has a slightly different flow topology. Two LEV's are formed, one at the beginning of the stroke that detaches as the pitching angle increases further, the second one is formed when the pitch angles starts to increase again. Similarly as for HM1 there are two TEV's per half cycle. The sine motion starts out stalled and then forms one large LEV. There is only one TEV, starting vortex, at the beginning of the stroke. Clearly, the vortical flow evolution is unique to all three cases, as was the case for the force history.

\section{Acknowledgments}

This work has been supported in part by the Air Force Office of Scientific Research's Multidisciplinary University Research Initiative.

\section{References}

\footnotetext{
${ }^{1}$ Hikaru Aono, Wei Shyy, and Hao Liu. Near wake vortex dynamics of a hovering hawkmoth. Acta Mech Sin, 25:23-26, 2009.

${ }^{2}$ Yeon Sik Baik, Jonathan M. Rausch, Luis P. Bernal, and Michael V. Ol. Experimental investigation of pitching and plunging airfoils at reynolds number between $1 \times 10^{4}$ and $6 \times 10^{4}$. AIAA 2009-4030, 2009 .

${ }^{3}$ James M. Birch and Michael H. Dickinson. The influence of wing-wake interaction on the production of aerodynamic forces in flapping flight. The journal of Experimental Biology, 206:2257-2272, 2003.

${ }^{4}$ Charles P. Ellingtion, Coen van den Berg, Alexander P. Willmott, and Adrian L.R. Thomas. Leading-edge vortices in insect flight. Nature, 384:626-630, 1996.

${ }^{5}$ Kenneth Granlund, Michael Ol, luis Bernal, and Steve Kast. Experiments on free-to-pivot hover motions of flat plates. AIAA 2010-4456, 2010.

${ }^{6}$ Hao Liu, Charles P. Ellington, Keiji Kawachi, Coen van den Berg, and Alexander P. Wellmott. A computational fluid dynamic study of hawkmoth hovering. Journal of experimental biology, 201:461-477, 1998.

${ }^{7}$ Barnes W. McCormick. Aerodynamics, Aeronautics, and Flight Mechanics. Wiley, 1994.

${ }^{8}$ C. Morrison, C.E.S. Cesnik, Luis P. Bernal, R. Vandenheede, and D. Kumar. Force measurements of a flapping wing with two angular degrees of freedom and bio-inspired kinematics. AIAA, Submitted for publishing, 2012.

${ }^{9}$ Jonathan M. Rausch, Yeon Sik Baik, Luis P. Bernal, Carlos E.S. Cesnik, Wei Shyy, and Michael V. Ol. Fluid dynamics of flapping rigid and spanwise-flexible elliptical flat plates at low reynolds numbers. AIAA 2010-4739.
} 
${ }^{10}$ Wei Shyy, Yongsheng Lian, Jian Tang, Dragos Viieru, and Hao Liu. Aerodynamics of low Reynolds number flyers. Cambridge University Press, 2008.

${ }^{11}$ Alexander P. Willmott and Charles P. Ellingtion. The mechanics of flight in the hawkmoth manduca sexta. The journal of Experimental Biology, 200:2705-2722, 1997.

${ }^{12}$ W.Shyy, H. Aono, S.K. Chimakurthi, P. Trizila, C.-K Kang, C.E.S. Cesnik, and H. Liu. Recent progress in flapping wing aerodynamics and aeroelasticity. Progress in Aerospace Sciences, 46:284-327, 2010.

${ }^{13}$ Derrick Yeo, Ella M. Atkins, Luis P. Bernal, and Wei Shyy. Experimental investigation of the pressure, force, and torque characteristics of a rigid flapping wing. AIAA, Submitted for publishing, 2012.

\section{8 of 18}

